\title{
Creeping waves in the shadow region with the Dirichlet and Neumann conditions
}

\author{
Anna Kirpichnikova \\ Division of Computing Science and Mathematics, University of Stirling, UK; \\ e-mail: anya@cs.stir.ac.uk \\ Natalia Kirpichnikova \\ Saint-Petersburg Department of Steklov's Mathematical Institute, Russia; \\ e-mail: nkirp@pdmi.ras.ru
}

\begin{abstract}
We construct shadow creeping waves in the problem of a plane wave diffraction by a smooth axially symmetric prolate body of revolution for both Dirichlet and Neumann boundary conditions. Using Fock's asymptotics as the initial data for the creeping wave amplitude, the theory of residues allows us to present the wave field in the main approximation.
\end{abstract}

\section{INTRODUCTION}

This paper is a continuation of the series of works on constructing the diffracted field in the vicinity of the axially symmetric prolate body of revolution from the plane wave propagating along the axis of revolution. The history of this problem goes back to V. A. Fock, who constructed the wave field in the main approximation in zone 2 (see Fig. 1), which is now called the "Fock's region" [1]. V. M. Babich and N. Ya. Kirpichnikova have developed a scheme to construct further terms of the approximation by using Leontovich-Fock parabolic equation method [2]. This problem was considered by I. V. Andronov $[3,4]$, who used methods different to those applied by the authors. N. Ya. Kirpichnikova and M. M. Popov described the diffracted field for the problem with the Dirichlet boundary conditions [5,6]. A. S. Kirpichnikova and N. Ya. Kirpichnikova obtained the first three terms of the wave field approximation for the problem with Neumann boundary conditions $[7,8]$. The novelty of the present paper is in the description of the creeping waves in the shadow region for the Neumann problem.

\section{Problem STATEMENT AND ANALYSIS}

We investigate diffraction of a plane wave $e^{i k z}$ incident onto the strictly convex smooth prolate body of revolution. The latter body will be referred to as a scatterer. Here $k=\frac{2 \pi}{\lambda}$ is a wave number, $\lambda$ is a wavelength of the incident wave. We assume that the geometric characteristics of the scatterer are much larger than the wavelength $\lambda$, and the axis of revolution coincides with $z$-axis.

The wave field satisfies Helmholtz equation outside of the scatterer together with either Dirichlet or Neumann boundary conditions on its surface and the limiting absorption principle far from the scatterer (see ch. 6, $§ 5[9]$ ).

The wave field is determined by the ray formulae in the illuminated area. In the vicinity of $s=0$, which belongs to the boundary of the geometric shadow, we introduce Fock's surface boundary layer $O\left(s k^{\frac{1}{3}}\right)=O(1), O\left(n k^{\frac{2}{3}}\right)=O(1)$, (see Fig. 1). Here $s$ is an arc length along the geodesic at the surface of the scatterer, $n$ measures the distance along the normal to the scatterer.

The ray method fails in Fock's zone 2 (see Fig. 1). The solution there is constructed by the Leontovich-Fock parabolic equation method, see $[1,5,7]$. The first three terms of the following expansion were constructed in the boundary layer 2 for both Dirichlet and Neumann problems $([5,7])$ :

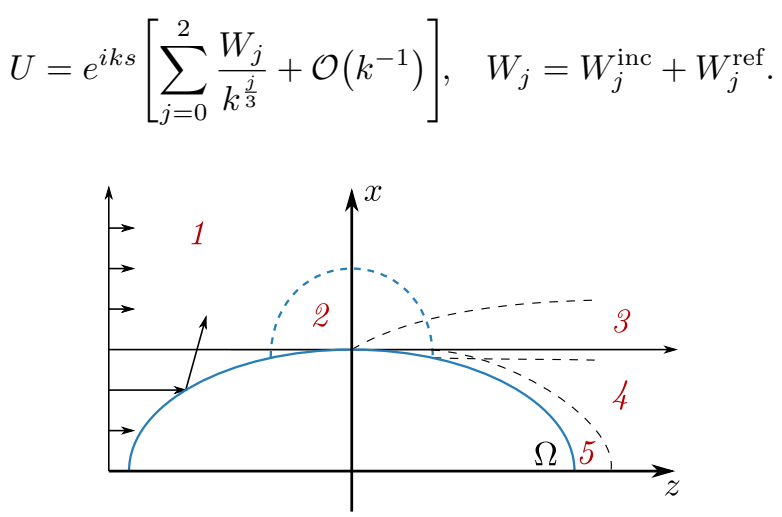

Figure 1: The main five zones near $\partial \Omega$ : lit zone 1, the Fock's zone 2, neighborhood of the limit ray 3 , zone 4 is a shadow, zone 5 is a surface layer full of creeping waves.

(C) 2019 IEEE. Personal use of this material is permitted. Permission from IEEE must be obtained for all other uses, in any current or future media, including reprinting/ republishing this material for advertising or promotional purposes, creating new collective works, for resale or redistribution to servers or lists, or reuse of any copyrighted component of this work in other works. 
To this end, the stretched dimensionless coordinates

$$
\sigma=2^{-\frac{1}{3}} \rho_{0}^{-\frac{2}{3}} k^{\frac{1}{3}} s, \quad \nu=2^{\frac{1}{3}} \rho_{0}^{-\frac{1}{3}} k^{\frac{2}{3}} n
$$

were introduced. Here $\rho_{0}$ is the curvature radius of the geodesic at the surface of the scatterer at point $s=0$.

The attenuation functions $W_{j}^{\text {inc }}(\sigma, \nu), j=0,1,2$, are constructed from the incident wave in coordinates $(\sigma, \nu)$. These functions have the form of contour integrals of a linear combination of Airy functions $v(t)$ and its derivatives $v^{\prime}(t)$ and polynomials on the dimensionless variable $\nu$ of order $2 j$ at $v(t)$ and of order $2 j-1$ at $v^{\prime}(t)$.

Functions $W_{j}^{\text {ref }}, j=0,1,2$, are shaped similar to functions $W_{j}^{\text {inc }}$, where instead of Airy functions $v(t), v^{\prime}(t)$ one should take Airy functions $w_{1}(t)$ and $w_{1}^{\prime}(t)$. They satisfy the limiting absorption principle as $\nu \rightarrow \infty$. Besides that, functions $W_{j}^{\text {ref }}, j=0,1,2$, satisfy the following recurrent system of differential equations

$$
\begin{gathered}
\mathcal{L}_{0} W_{0}^{\text {ref }}=0, \quad \mathcal{L}_{0} W_{1}^{\text {ref }}+\mathcal{L}_{1} W_{0}^{\text {ref }}=0 \\
\mathcal{L}_{0} W_{2}^{\text {ref }}+\mathcal{L}_{1} W_{1}^{\text {ref }}+\mathcal{L}_{2} W_{0}^{\text {ref }}=0, \quad \text { and so on. }
\end{gathered}
$$

The main term of the asymptotic expansion of the total wave field in this problem is often called the "famous V.A. Fock's formula". This solution has been constructed in the vicinity of $s=0$, i.e. at the point where the incident ray touches the scatterer. Let us present here those famous formulae for the Dirichlet problem:

$$
\begin{aligned}
& U_{0}^{\mathrm{Dir}}=e^{i k s}\left(W_{0}^{\mathrm{inc}}+W_{0}^{\mathrm{ref}}\right) \\
& =\frac{e^{i k s}}{\sqrt{\pi}} \int_{\Gamma} e^{i \sigma \zeta}\left[v(\zeta-\nu)-\frac{v(\zeta)}{w_{1}(\zeta)} w_{1}(\zeta-\nu)\right] d \zeta
\end{aligned}
$$

and for the Neumann problem:

$$
U_{0}^{\mathrm{Neu}}=\frac{e^{i k s}}{\sqrt{\pi}} \int_{\Gamma} e^{i \sigma \zeta}\left[v(\zeta-\nu)-\frac{v^{\prime}(\zeta)}{w_{1}^{\prime}(\zeta)} w_{1}(\zeta-\nu)\right] d \zeta
$$

Contour $\Gamma$ in formulae (2), (3) goes along the ray $\arg (\zeta)=\frac{2 \pi}{3}$ from infinity to zero, then along the ray $\arg (\zeta)^{3}=0$ from zero to infinity. Contour $\Gamma$ envelopes the line of roots of Airy functions $w_{1}(t), w_{1}^{\prime}(t)$, i.e. the ray $\arg (t)=\frac{\pi}{3}$.

The incident plane wave field does not reach the shadowed zone 5 near the scatterer surface. However, following the observation point staying in the boundary layer with respect to $\nu$, i.e. $\nu=\mathcal{O}(1)$, it enters the shadow zone 5 while $s>0$ is increasing. In the local stretched coordinates $(\sigma, \nu)$, those conditions take form of growing $\sigma \rightarrow+\infty$ and bounded $\nu=\mathcal{O}(1)$. In the upper half-plane, where the imaginary part of $\zeta$ is positive as $\sigma \rightarrow+\infty$, the exponent $e^{i \sigma \zeta}$ decays as fast as $\exp (-\sigma \operatorname{Im} \zeta)$. Lifting up the integration contour into the upper half-plane allows us to calculate a number of residues, which appear because we leave out the singularities of $w_{1}\left(\zeta_{p}\right)$ for the Dirichlet problem, and the singularities of $w_{1}^{\prime}\left(\zeta_{p}^{\prime}\right)$ for the Neumann problem.

Formulae (2), (3) result in the following simplified version in the shadow zone 5 once integrals are replaced with the sums:

$$
U_{0}^{\text {Dir }} \asymp e^{i k s}(-2 \sqrt{\pi} i) \sum_{p=1}^{N} e^{i \sigma \zeta_{p}} \frac{w_{1}\left(\zeta_{p}-\nu\right)}{\left[w_{1}^{\prime}\left(\zeta_{p}\right)\right]^{2}}
$$

for the Dirichlet problem and

$$
U_{0}^{\mathrm{Neu}} \asymp e^{i k s}(2 \sqrt{\pi} i) \sum_{p=1}^{N} e^{i \sigma \zeta_{p}^{\prime}} \frac{w_{1}\left(\zeta_{p}^{\prime}-\nu\right)}{\zeta_{p}^{\prime}\left[w_{1}\left(\zeta_{p}^{\prime}\right)\right]^{2}}
$$

for the Neumann problem.

Since $e^{i \sigma \zeta}$ decays fast as $\sigma \rightarrow+\infty$, we restrict (2), (3) by the first root of Airy function and its derivative $\zeta_{1}, \zeta_{1}^{\prime}$, the resulting solutions will be denoted by $U_{01}^{\mathrm{Dir}}(\sigma, \nu)$ and $U_{01}^{\mathrm{Neu}}(\sigma, \nu)$.

Let us now construct the creeping wave in the shadow region 5 (see $[2,9,10])$. We choose the following form for the creeping wave in the boundary layer $\nu(s, n)=\mathcal{O}(1)$ and $s=\mathcal{O}(1)$ (see [12]):

$$
U=e^{i k s+i k^{\frac{1}{3}} \phi(s)} \sum_{m=0}^{\infty} V_{m}(s, \nu) k^{-\frac{m}{3}}
$$

where

$$
\phi(s)=2^{-\frac{1}{3}} \zeta \int_{0}^{s} \rho^{-\frac{2}{3}}(s) d s .
$$

Separation constant $\zeta$ appears to be equal to the root of Airy function $w_{1}\left(\zeta_{p}\right)=0$ for the Dirichlet problem and to the root of its derivative for the Neumann problem, i.e. $w_{1}^{\prime}\left(\zeta_{p}^{\prime}\right)=0$.

Note that in contrast with region 2 , the arc length $s$ in region 5 is of order $s=\mathcal{O}(1)$, whereas the normal $\nu(s, n)$ is defined in the following way (cp. formula (1)):

$$
\nu(s, n)=2^{\frac{1}{3}} \rho^{-\frac{1}{3}}(s) k^{\frac{2}{3}} n, \quad \nu(s, n)=\mathcal{O}(1) .
$$

The Fock's parameter $\boldsymbol{M}$ is now large, depends on $s$ and equals to

$$
\boldsymbol{M}(s)=\left(\frac{k \rho(s)}{2}\right)^{\frac{1}{3}} .
$$


Formulae (6) and (7) show that when the wave propagates along the scatterer, the creeping wave decays as exponent $\exp \left\{i 2^{-\frac{1}{3}} k^{\frac{1}{3}} \zeta \int_{0}^{s} \rho^{-\frac{2}{3}}(s) d s\right\}$ for which $\arg (\zeta)$ equals $\frac{\pi}{3}$, with $s>0$. Physically, this is explained by the fact that when the creeping waves propagate, the rays coming down tangentially to the boundary of the scatterer carry away the energy from the boundary layer 5 (see Fig. 2, [11], [9, ch. 12]).

We obtain a recurrent system of equations from the Helmholtz equation for functions $V_{m}(s, \nu)$. The solution is found in the form (6), where $\phi(s)$ is in the form (7). After the substitution into the Helmholtz equation, we equate the coefficients at the same powers of $k$. We assume that $\nu$ is a function of both variables $s$ and $n$, since we are interested in the solution in region 5 . We have

$$
\sum_{m=0}^{j} L_{m} V_{j-m}=0, \quad j=0,1,2, \ldots
$$

where

$$
\begin{aligned}
L_{0}= & \frac{\partial^{2}}{\partial \nu^{2}}+(\nu-\zeta) \\
L_{1}= & \frac{\rho i}{2}\left(\frac{2}{\rho}\right)^{\frac{1}{3}}\left(2 \frac{\partial}{\partial s}+\frac{\partial \ln f}{\partial s}\right) ; \\
L_{2}= & \left(\frac{2}{\rho}\right)^{\frac{2}{3}}\left[\left(-\frac{\zeta^{2}}{4}+\nu \zeta-\frac{3 \nu^{2}}{4}\right)+\frac{1}{2}(1+\boldsymbol{\Lambda}) \frac{\partial}{\partial \nu}\right] ; \\
L_{3}= & i\left[\left(\frac{\zeta}{2}-\nu\right)\left(2 \frac{\partial}{\partial s}+\frac{\partial \ln f}{\partial s}\right)-\frac{\zeta \rho^{\prime}}{3 \rho}\right. \\
& \left.+\frac{\nu}{2} \frac{\partial}{\partial s} \ln (f \rho)-\frac{\nu \boldsymbol{\Lambda}}{2} \frac{\partial}{\partial s} \ln (f)\right]
\end{aligned}
$$

In the latter formulae for $L_{2}$ and $L_{3}$, there appears the large parameter $\boldsymbol{\Lambda}=\frac{\rho(s)}{f(s)}$, which characterizes the elongation of th body, $f(s)$ is the principal radius of curvature of the scatterer's surface along a parallel, and $\rho(s)$ is the principal radius of curvature along a meridian.

The following analysis is based on the classical parabolic equation method, but appears as a two-scale asymptotic expansion with respect to two large independent parameters: $\boldsymbol{M}(s)$ and $\boldsymbol{\Lambda}(s)$. This two-scale expansion allows us to obtain formulae for calculating the wave field, in particular, the current on the surface of the scatter at different ratios between $\boldsymbol{M}(s)$ and $\boldsymbol{\Lambda}(s)$. It can be shown that the recurrent system of equations (8) will retain its asymptotic character at $\boldsymbol{M}(s)$ provided that $\boldsymbol{\Lambda}(s)=\boldsymbol{M}^{2-\varepsilon}$ for $0<\varepsilon<2$.

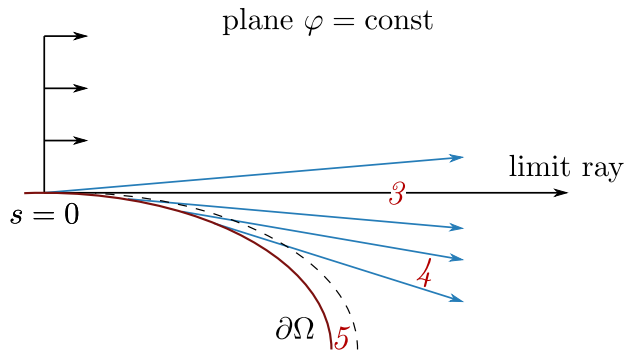

Figure 2: Penumbra and shadow regions $\sigma>0$. The field in the shadow region 4 between regions 3 and 5 has ray nature of the leaving wave. Region 5 is the creeping waves zone with $\nu=\mathcal{O}(1), s=\mathcal{O}(1)$.

We use the following scheme to solve system (8). Operator $L_{0}$ is the Airy operator and with homogeneous boundary conditions and it defines the following problem for $V_{0}$ :

$$
\left\{\begin{array}{l}
\frac{\partial^{2}}{\partial \nu^{2}} V_{0}+(\nu-\zeta) V_{0}=0 \\
\left.V_{0}\right|_{\nu=0}=0 \quad\left(\left.\frac{\partial V_{0}}{\partial \nu}\right|_{\nu=0}=0\right) \\
V_{0} \underset{\nu \rightarrow+\infty+i \epsilon}{\longrightarrow} 0, \quad \epsilon>0 .
\end{array}\right.
$$

The latter limit agrees with the principle of limiting absorption; see [10].

Problem (9) has the following non-trivial solution

$$
V_{0}=V_{0, p}=A_{0}(s) \cdot w_{1}(\zeta-\nu),
$$

where

$\zeta= \begin{cases}\zeta_{p}, & \text { for the Dirichlet problem, } w_{1}\left(\zeta_{p}\right)=0 \\ \zeta_{p}^{\prime}, & \text { for the Neumann problem, } w_{1}^{\prime}\left(\zeta_{p}^{\prime}\right)=0\end{cases}$

and function $A(s)$ is arbitrary at this stage. The function $w_{1}(\zeta-\nu)$ has asymptotics as $\nu \rightarrow+\infty$ :

$$
w_{1}(-\nu) \sim \nu^{-\frac{1}{4}} \exp \left\{\frac{i \pi}{4}+\frac{2 i \nu^{\frac{3}{2}}}{3}\right\} \text {. }
$$

System (8) implies the following recurrent sequence of Sturm-Liouville problems

$$
\left\{\begin{array}{l}
L_{0} V_{j}+L_{1} V_{j-1}+\ldots+L_{j} V_{0}=0, \\
\left.V_{j}\right|_{\nu=0}=0, \quad\left(\left.\frac{\partial V_{j}}{\partial \nu}\right|_{\nu=0}=0\right), \\
V_{j} \underset{\nu \rightarrow+\infty+i \epsilon}{\longrightarrow} 0, \quad \epsilon>0, \quad j=0,1,2, \ldots
\end{array}\right.
$$


Equations (11) are considered on the Cartesian product of the surface area of the scatterer and a ray $0 \leq \nu \leq+\infty$.

Assume $j=1$, then (11) takes the form a nonhomogeneous Sturm-Liouville problem:

$$
\left\{\begin{array}{l}
L_{0} V_{1}=-L_{1} V_{0}, \\
\left.V_{1}\right|_{\nu=0}=0, \quad\left(\left.\frac{\partial V_{1}}{\partial \nu}\right|_{\nu=0}=0\right), \\
V_{1} \underset{\nu \rightarrow+\infty+i \epsilon}{\longrightarrow} 0, \quad \epsilon>0 .
\end{array}\right.
$$

The corresponding homogeneous problem has a non-trivial solution. For the solvability of problem (12), it is necessary and sufficient that the free term $\left(-L_{1} V_{0}\right)$ is orthogonal to the solution (10) of the corresponding homogeneous problem, namely,

$$
\int_{0}^{e^{i \frac{\pi}{3}} \infty}\left(2 \frac{\partial V_{0}}{\partial s}+V_{0} \frac{\partial \ln f}{\partial s}\right) \cdot w_{1}(\zeta-\nu) d \nu=0 .
$$

The transport equation for the amplitude $A_{0}$ will be considered in more detail, given that $V_{0}=A_{0} w_{1}(\zeta-\nu)$ :

$$
\begin{aligned}
& \int_{0}^{e^{i \frac{\pi}{3}} \infty}\left(A_{0} \frac{\partial \ln f}{\partial s} w_{1}^{2}(\zeta-\nu)+2 \frac{d A_{0}}{d s} w_{1}^{2}(\zeta-\nu)\right) d \nu \\
& +\frac{2}{3} \frac{\rho^{\prime}}{\rho} A_{0} \int_{0}^{e^{i \frac{\pi}{3}} \infty} \nu w_{1}(\zeta-\nu) \cdot w_{1}^{\prime}(\zeta-\nu) d \nu=0
\end{aligned}
$$

The last term is derived from the following equality: $\frac{\partial}{\partial s} w_{1}(\zeta-\nu)=-w_{1}^{\prime}(\zeta-\nu) \frac{\partial \nu}{\partial s}=\frac{\nu}{3} \frac{\partial \ln \rho}{\partial s} w_{1}^{\prime}(\zeta-\nu)$.

The corresponding integral is equal to

$$
\begin{aligned}
& \int_{0}^{e^{i \frac{\pi}{3}} \infty} \nu w_{1}(\zeta-\nu) w_{1}^{\prime}(\zeta-\nu) d \nu \\
& =\frac{1}{2} \int_{0}^{e^{i \frac{\pi}{3}} \infty} w_{1}^{2}(\zeta-\nu) d \nu \\
& =\frac{1}{2}\left\{\begin{array}{ll}
-\left[w_{1}^{\prime}(\zeta)\right]^{2}, & \text { where } \zeta=\zeta_{p} \\
\zeta\left[w_{1}(\zeta)\right]^{2}, & \text { for the Dirichlet problem, } \zeta=\zeta_{p}^{\prime}
\end{array}\right. \text { for the Neumann problem. }
\end{aligned}
$$

Finally, the transport equation for $A_{0}$ is

$$
\left[2 \frac{d A_{0}}{d s}+A_{0} \frac{d \ln f}{d s}+\frac{A_{0}}{3} \frac{d \ln \rho}{d s}\right]=0 .
$$

Function $A_{0}(s)$ equals

$$
A_{0}(s)=A_{0}(0) \frac{1}{\sqrt{f(s)}} \frac{1}{\sqrt[6]{\rho(s)}} .
$$

Coefficients $A_{0}(0)$ will be chosen by finding residues (4), (5) for $W_{0}^{\text {ref }}(\sigma, \nu)$ in the vicinity of the point $s=0$ (see [7]), resulting in

$$
\begin{aligned}
A_{0, p}^{\mathrm{Dir}}(0) & =-\frac{2 \sqrt{\pi} i \sqrt[6]{\rho_{0}} \sqrt{f(0)}}{\left[w_{1}^{\prime}\left(\zeta_{p}\right)\right]^{2}}, \\
A_{0, p}^{\mathrm{Neu}}(0) & =\frac{2 \sqrt{\pi} i \sqrt[6]{\rho_{0}} \sqrt{f(0)}}{\zeta_{p}^{\prime}\left[w_{1}\left(\zeta_{p}^{\prime}\right)\right]^{2}}
\end{aligned}
$$

We supplied the equation for $A_{0}(s)$ (13) with the initial value $A_{0}(0)$, i.e. for the Dirichlet problem we use formula (14), and for the Neumann problem we use formula (15). These initial conditions arise from the requirement of "gluing" creeping waves with the solution for the reflected wave in the shadow part of the Fock's area $(s>0)$.

Now we come to finding $V_{1}$ from (12). We choose in a particular solution of the inhomogeneous equation $L_{0} V_{1}=-L_{1} V_{0}$ being polynomials at $\nu$ of the following kind:

$$
P_{1}=P_{1}^{(2)} \nu^{2}+P_{1}^{(1)} \nu ; \quad Q_{1}=Q_{1}^{(1)} \nu+Q_{1}^{(0)},
$$

multiplyed by the Airy function $w_{1}(\zeta-\nu)$. The polynomials are defined uniquely. If we substitute function $\alpha_{1}(s)$ by the formula $A_{1}(s)=A_{0}(s) \alpha_{1}(s)$, the solution of the problem (12) becomes

$$
V_{1}(s, \nu)=A_{0}(s) w_{1}(\zeta-\nu)\left[\alpha_{1}(s)+\frac{i \rho^{\prime}}{12}\left(\frac{2}{\rho}\right)^{\frac{1}{3}} \nu^{2}\right]
$$

If the observation point $M(s, \nu)$, which follows the creeping wave, goes beyond the boundary layer $\left(n \sim \mathcal{O}\left(k^{-\frac{2}{3}}\right)\right)$ moving away from the surface of the scatterer, then the value of $\nu$ will become much larger than one, and the Airy's function $w_{1}$ can be replaced with its asymptotics at $\nu \rightarrow+\infty$. Then the most oscillating factor in the creeping wave's phase will be as follows:

$$
\exp \left(i k s+i \frac{2}{3} \nu^{\frac{3}{2}}+i \frac{\pi}{4}+i \frac{k^{\frac{1}{3}} \zeta}{2^{\frac{1}{3}}} \int_{0}^{s} \frac{d s}{\rho^{\frac{2}{3}}(s)}-i \zeta \sqrt{\nu}\right)
$$

\section{Conclusions}

Thus, at a distance from the surface of the scatterer, the radiation field of the creeping wave (at least in the first approximation) is a set of beams corresponding to the outgoing wave having the surface of the scatterer as a caustic; see Fig. 2. 
The third term in the exponent of the creeping wave (16) for $\zeta=\zeta_{p},\left(w_{1}\left(\zeta_{p}\right)=0\right)$ or $\zeta=$ $\zeta_{p}^{\prime},\left(w_{1}^{\prime}\left(\zeta_{p}^{\prime}\right)=0\right)$ for $\arg \left(\zeta_{p}\right)\left(\arg \left(\zeta_{p}^{\prime}\right)\right)=\frac{\pi}{3}$ and $s>0$ decrease exponentially at a distance from the surface of the scatterer. Physically, this happens because when the creeping wave propagates, its rays, spreading along the tangent to the surface of the scatterer, carry the energy away from the surface layer (see [11] and [9]).

The elongation parameter $\boldsymbol{\Lambda}$ in the operator $L_{2}$ influences the transport equations for $A_{1}(s)$ only for the Neumann case. Creeping waves obtained in this paper give us an example of diffraction in its original narrow sense as a wave goes around the obstacles. The creeping rays, falling off in the tangential to the obstacle direction, generate Friedlander-Keller's diffracted ray field in the shadow region [12].

\section{ACKNOWLEDGEMENT}

N. Kirpichnikova has been partially supported by the Russian Foundation for Basic Research (RFBR) grant 17-01-00529A.

\section{REFERENCES}

[1] Fock, V.A., 1965, Electromagnetic Diffraction and Propagation Problems, International Series of Monographs on Electromagnetic Waves, Vol. 1, Pergamon Press, Oxford, London, Edinburgh, New York, Paris, Frankfurt.

[2] Babich, V. M., Kirpichnikova, N. Y., 1979, The Boundary-Layer Method in Diffraction Problems, Springer-Verlag, Berlin, Heidelberg, New York.

[3] Andronov, I. V., 2011, Diffraction of strongly elongation body of revolution, Acoustic Physics, Vol. 57(2), pp. 121-126.

[4] Andronov, I. V., 2012, Diffraction of a plane wave incident at a small angle to the axis of a strongly elongation spheroid, Acoustic Physics, Vol. 58(5), pp. 521-529.

[5] Kirpichnikova, N. Ya., Popov, M. M., 2013, Leontovich-Fock parabolic equation method in the problems of short-wave diffraction by prolate bodies, Journal of Mathematical Sciences, Vol. 194(42), pp. 30-43.

[6] Popov, M. M., Kirpichnikova, N. Ya., 2014, On problems of applying the parabolic equation to diffraction by prolate bodies, Acoustical Physics, Vol. 60(4), pp. 363-370.

[7] Kirpichnikova, N. Ya., Kirpichnikova, A.S., 2019, Leontovich-Fock parabolic equation method in the neumann diffraction problem on a prolate body of revolution, Journal of Mathematical Sciences, Vol. 238(5), pp. 658-675.

[8] Kirpichnikova, A., Kirpichnikova, N., 2018, Comparison of the currents in the Dirichlet and Neumann shortwave diffraction problems of a plane wave from smooth prolate bodies of revolution, Proceedings of the International Conference "Days on Diffraction 2018", IEEE, pp. $161-167$.

[9] Babich, V.M., Buldyrev, V.S., 2009, Asymptotic Methods in Short-Wavelength Diffraction Theory, Alpha Science, Oxford.

[10] Babich, V.M., Kiselev, A.P., 2018, Elastic Waves: High Frequency Theory, Chapman \& Hall/CRC Monographs and Research Notes in Mathematics, CRC Press, New York.

[11] Keller, J. B., 1956, Diffraction of a convex cylinder, IRE Transactions on Antennas and Propagation, Vol. 4(3), pp. 312-321.

[12] Friedlander, F. G., Keller, J. B., 1955, Asymptotic expansions of solutions of $\left(\nabla^{2}+k^{2}\right) u=0$, Communications on Pure and Applied Mathematics, Vol. 8(3), pp. 378-394. 